ISSN 0103-8478

\title{
Biologia de Mahanarva fimbriolata em gramíneas forrageiras
}

\author{
Biology of Mahanarva fimbriolata on forage grasses
}

\author{
Eliane Grisoto ${ }^{\mathrm{I}}$ José Djair Vendramim ${ }^{\mathrm{I}}$ André Luiz Lourenção ${ }^{\mathrm{II}}$ \\ José Alfredo Usberti Filho ${ }^{\mathrm{II}}$ Carlos Tadeu dos Santos Dias ${ }^{\mathrm{I}}$
}

\section{RESUMO}

\begin{abstract}
Estudou-se a biologia de Mahanarva fimbriolata nas gramineas Setaria anceps $c v$. 'Kazungula', Brachiaria brizantha $c v$. 'Marandu', ecótipos $B B$ 33, BB 39, seleções IACBBS 5 e IAC-BBS 8, e cana-de-açúcar. 'SP-80-1842' (testemunha) em laboratório, visando a avaliar a resistência desses genótipos a essa praga. Os parâmetros avaliados foram: mortalidade e duração da fase de ninfa, razão sexual, periodo de préoviposição, número de ovos por fêmea, longevidade, duração e viabilidade da fase embrionária. Constatou-se maior mortalidade e prolongamento na fase de ninfa em IAC-BBS 8, B. brizantha cv. 'Marandu'e S. anceps $c v$. 'Kazungula'. Os menores valores de longevidade e fecundidade foram verificados em IAC-BBS 8 e $I A C$-BBS 5 e os maiores em cana-de-açucar. A menor viabilidade da fase embrionária foi obtida em IAC-BBS 5 e a maior em IAC$B B S$ 8. Segundo os resultados da tabela de vida, a taxa líquida de reprodução (Ro) e a taxa finita de crescimento ( $\lambda$ ) foram menores em IAC-BBS 8. Concluiu-se que IAC-BBS 8 é o genótipo mais resistente a $\boldsymbol{M}$. fimbriolata, sendo a resistência do tipo antibiose e/ ou não-preferência para alimentação.
\end{abstract}

Palavras-chave: Cercopidae, resistência de plantas a insetos, cigarrinha-das-raizes.

\section{ABSTRACT}

Under controlled conditions, six forage grasses (Setaria anceps $c v$. 'Kazungula' and Brachiaria brizantha $c v$. 'Marandu'; and codified genotypes BB33, BB39; BBS 5 and BBS8), along with a control check (sugarcane cv. 'SP80-1842') were evaluated as to Mahanarva fimbriolata resistance. The following parameters have been evaluated: nymphal phase duration and mortality, sex ratio, pre-oviposition period, number of eggs per female, longevity, viability and embryonic period. The highest mortalities and longer nymphal periods occurred on BBS 8, cv. 'Marandu' and cv. 'Kazungula'. BBS5 also caused high nymph mortality, however with the shortest nymphal period. The lowest longevity and fecundity values were detected for BBS5 and $B B S 8$, whereas the sugarcane control check revealed the highest one. As to embryonic phase viability, BBS 8 originated the highest values and BBS 5, the lowest ones. Among the tested plants, BBS 8 along with the $\mathrm{cv}$. 'Marandu', proved to be the most resistant genetic material, both through antibiosis and/or nonpreference for feeding mechanism.

Key words: Cercopidae, host plant resistance, Gramineae, spittlebug.

\section{INTRODUÇÃO}

A produção de gado de corte brasileira caracteriza-se pela exploração extensiva, tendo as pastagens como base de alimentação do rebanho. Nas últimas décadas, houve grande introdução de gramíneas, principalmente do gênero Brachiaria, extensivamente plantadas devido à excelente produtividade e adaptabilidade a solos ácidos e com baixa fertilidade (LAPOINTE et al.,1989).

Um dos fatores limitantes para boa produtividade dessas gramíneas tem sido os danos causados pelo complexo de cigarrinha-das-pastagens (Hemiptera: Cercopidae) tanto na fase de ninfa como de adulto (FERRUFINO \& LAPOINTE, 1989). As ninfas se alimentam nas raízes, inserindo seus estiletes nos vasos do xilema e sugando a seiva bruta, destruindo, dessa forma, os vasos condutores, o que dificulta o transporte de água e nutrientes, debilitando a planta e causando o sintoma conhecido por desordem fisiológica (BYERS \& WELLS, 1966).

'Escola Superior de Agricultura "Luiz de Queiroz" (ESALQ), Universidade de São Paulo (USP), Piracicaba, SP, Brasil.

"Instituto Agronômico de Campinas (IAC), Av. Barão de Itapura, 1481, CP 28, 13012-970, Campinas, SP, Brasil. E-mail: andre@iac.sp.gov.br.

*Autor para correspondência. 
No entanto, o principal dano é causado pelos adultos que, ao se alimentarem, injetam toxinas presentes na saliva, causando a "queima" da folha, reduzindo sensivelmente a capacidade fotossintética da planta (VALÉRIO, 1985).

Algumas cultivares consideradas resistentes às principais espécies de cigarrinha-daspastagens vêm se mostrando suscetíveis a Mahanarva fimbriolata (Stål), como é o caso de B. brizantha cv. 'Marandu'. M. fimbriolata apresenta tamanho maior, quando comparada às outras espécies de cigarrinhadas-pastagens, como Notozulia entreriana (Berg), sendo observado em campo danos mais intensos para a primeira espécie (VALÉRIO, 2006). O controle químico é considerado economicamente inviável por serem as pastagens culturas de baixo valor por unidade de área. Entre os métodos alternativos, destaca-se a utilização de plantas resistentes que, sob o ponto de vista econômico e ambiental, é a ferramenta mais indicada para essas situações, podendo ser utilizada juntamente com o controle biológico e o cultural, além de ser de baixo custo e de fácil adoção por parte dos produtores, uma vez que o controle é feito na aquisição das sementes, contribuindo para o manejo integrado dessa praga (NILAKHE, 1987; VALÉRIO, 2006). Assim, o presente trabalho teve como objetivo avaliar a biologia de $\mathbf{M}$. fimbriolata em sete genótipos de gramíneas, visando a identificar genótipos resistentes à referida praga.

\section{MATERIAL E MÉTODOS}

A pesquisa foi desenvolvida em condições de laboratório $\left(25 \pm 1^{\circ} \mathrm{C}\right.$; UR $70 \pm 10 \%$ e fotofase de 14h). A criação estoque de $\boldsymbol{M}$. fimbriolata foi mantida segundo a técnica desenvolvida por GARCIA et al. (2007) sobre plantas de cana-de-açúcar cv. 'SP801842'. As espécies Setaria anceps, cv. 'Kazungula', e Brachiaria brizantha, cv. 'Marandu', foram fornecidas pelo Instituto de Zootecnia de Nova Odessa, enquanto as gramíneas BB 33, BB 39, IAC-BBS 5 e IAC-BBS 8, todas de B. brizantha, foram desenvolvidas e fornecidas pelo Instituto Agronômico (IAC). Visando a homogeneizar os tratamentos, todas as gramíneas foram replantadas na ESALQ, em casa de vegetação. Para avaliar o efeito das referidas gramíneas sobre a biologia de $\boldsymbol{M}$. fimbriolata, foi utilizada como testemunha a cana-deaçúcar cv. 'SP80-1842'. Para determinar a duração e a viabilidade do período de ninfa, foram utilizadas plantas com dois meses de idade, plantadas em recipientes de $200 \mathrm{ml}$. Antes da inoculação, cortouse o fundo dos copos, com a finalidade de expor as raízes. Foram inoculadas cinco ninfas recémeclodidas por planta, provenientes de criação estoque, sendo cada tratamento composto por dez repetições. Após a inoculação, as plantas foram encaixadas sobre tampas perfuradas de potes de $500 \mathrm{ml}$, isolando as ninfas e propiciando um microclima favorável para seu desenvolvimento. A mortalidade ninfal foi avaliada diariamente, caracterizada pela paralisação na produção de espuma (morte por dessecação). Após a emergência e a sexagem dos adultos, com base na policromia alar e na genitália, foram formados casais oriundos de cada tratamento. Cada casal foi colocado em uma gaiola cilíndrica de plástico transparente $(10 \mathrm{~cm} \varnothing \times 70 \mathrm{~cm}$ altura $)$, cada uma contendo uma muda do respectivo tratamento plantada em copo plástico de $200 \mathrm{ml}$. Na base dos copos, colocou-se um disco de algodão como substrato para oviposição. A contagem dos ovos foi feita diariamente. Foi verificada a fecundidade das fêmeas e a longevidade de ambos os sexos de $\boldsymbol{M}$. fimbriolata, nos diferentes genótipos. De maneira complementar, avaliou-se a viabilidade e a duração do período embrionário. Foram distribuídos até 50 ovos por placa de Petri, as quais foram forradas com papel filtro, umedecido diariamente.

$\mathrm{O}$ delineamento experimental foi $\mathrm{O}$ inteiramente casualizado, sendo os dados submetidos à análise de variância e as médias comparadas pelo teste de Tukey $(\mathrm{P} \leq 0,05)$, utilizando o software SAS. A análise de agrupamento foi feita pelo método não ponderado de agrupamento aos pares, utilizando médias aritméticas (UPGMA), o qual agrupa indivíduos de acordo com a similaridade. Para a análise, utilizaram-se os parâmetros duração e mortalidade de ninfa, razão sexual, longevidade de adultos, período de pré-oviposição, número de ovos por fêmea e duração e viabilidade da fase de ovo, por meio do programa Systat 3.0 (WILKINSON, 1992). Foram elaboradas tabelas de vida para cada genótipo, utilizando-se os parâmetros viabilidade de ovo a adulto, número de fêmeas adultas em cada genótipo, razão sexual, mortalidade diária de fêmeas, período de pré-oviposição e número médio de ovos por fêmea em cada data de oviposição, segundo SILVEIRA NETO et al. (1976). Os parâmetros da tabela de vida de fertilidade e respectivos erros padrão foram estimados por meio da técnica de Jackknife (MEYER et al., 1986) e as médias comparadas pelo teste " $t$ " unilateral $(\mathrm{P} \leq 0,05)$, utilizando o software 'Lifetable. sas' (MAIA et al., 2000) no ambiente "SAS System".

\section{RESULTADOS E DISCUSSÃO}

Fase de ninfa

O período ninfal de $\boldsymbol{M}$. fimbriolata observado em IAC-BBS 8 (39,7 dias) foi maior 
$(\mathrm{F}=7,68, \mathrm{P}<0,0001)$ que os valores encontrados em IAC-BBS 5 (em que foi registrado o menor valor, 34,5 dias), $\boldsymbol{S}$. anceps cv. 'Kazungula' (36,8 dias) e BB 33 (36,9 dias). Nos demais genótipos, incluindo a cana-de-açúcar, cv. 'SP80-1842', foram encontrados valores intermediários que variaram entre 38,2 e 39,2 dias e que não diferiram dos demais genótipos, com exceção de IAC-BBS 5 (Tabela 1). As maiores mortalidades das ninfas da cigarrinha foram registradas em $B$. brizantha cv. 'Marandu' $(66 \%)$, em IAC-BBS $8(64 \%)$ e em S. anceps cv. 'Kazungula' (62\%), cujos valores diferiram $(\mathrm{F}=10,81, \mathrm{P}<0,0001)$ dos encontrados em cana-deaçúcar (22\%) e nos genótipos BB 33 e BB 39 (26 e $28 \%$, respectivamente). O valor encontrado em IACBBS 5 (54\%) não diferiu dos registrados nos três genótipos que provocaram as maiores mortalidades e tampouco do observado em BB 39, um dos que propiciaram baixa mortalidade do inseto (Tabela 1). Parâmetros como duração e sobrevivência da fase de ninfa podem ser utilizados para verificar a qualidade nutricional da fonte de alimento, sendo que o alongamento do período ninfal pode estar associado com a menor sobrevivência, como observado neste trabalho em IAC-BBS 8 e $\boldsymbol{B}$. brizantha cv. 'Marandu'. Em S. anceps cv. 'Kazungula' e IACBBS 5, entretanto, em que foram verificadas menores durações, observaram-se altas mortalidades ninfais. É possível que nesses três genótipos tenha ocorrido uma seleção natural das ninfas de cigarrinha, de modo que tenham sobrevivido apenas as mais vigorosas e que, por isso, teriam completado mais rapidamente a sua fase de desenvolvimento. Em pesquisas desenvolvidas por FERRUFINO \& LAPOINTE (1989), foi observado prolongamento no tempo de desenvolvimento, aumento na mortalidade de ninfas e redução no peso de fêmeas de Zulia colombiana (Lallemand), quando criadas em B. brizantha, cv. 'Marandu', em comparação a 11 acessos do gênero Brachiaria e Andropogon gayanus CIAT 621. Altos valores de mortalidade na fase de ninfa podem estar associados ao processo de ecdise, como relatado por LAPOINTE et al. (1992) em B. jubata CIAT 16531, o que, entretanto, não foi observado neste trabalho. As ninfas criadas em S. anceps cv. 'Kazungula' e IAC-BBS 8 foram observadas produzindo espuma um dia após a inoculação, porém, nas avaliações seguintes, elas se encontravam quase ou totalmente sem espuma, sendo que muitas morreram por dessecação. Dados semelhantes foram relatados para Zulia entreriana (Berg), criada em B. brizantha cv. 'Marandu', Paspalum guenoarum e Paspalum plicatulum, quando as ninfas iniciaram a produção de espuma, mas cessaram essa produção um dia após a inoculação, morrendo dessecadas (NILAKHE, 1987). Nos outros genótipos do presente trabalho, a produção de espuma foi constante. Testando 30 genótipos de capim-elefante (Pennisetum purpureum) quanto à resistência a Mahanarva spectabilis (Distant), AUAD et al. (2007) registraram variação na sobrevivência de ninfas de 37,5 a 100\%. Avaliando o efeito de variedades de cana-de-açúcar sobre o desenvolvimento de $\boldsymbol{M}$. fimbriolata, GARCIA (2006) verificou variações no período de ninfa de 35 a 38 dias e na viabilidade de 50 a $78 \%$, dados semelhantes aos obtidos neste estudo para a cv. 'SP80-1842'.

\section{Fase adulta}

Em relação ao período médio de préoviposição, embora os valores tenham variado entre 9,0 dias (BBS 8) e 6,0 dias (BBS 5, B. brizantha, cv. 'Marandu', e cana-de-açúcar), não houve diferença

Tabela 1 - Médias ( \pm erro padrão) de duração e mortalidade da fase ninfal, e período de pré-oviposição e número de ovos por fêmea de Mahanarva fimbriolata criada em sete gramíneas $\left(\mathrm{T}=25 \pm 1^{\circ} \mathrm{C}\right.$; $\mathrm{UR}=70 \pm 10 \%$; fotofase $\left.14 \mathrm{~h}\right)$.

\begin{tabular}{|c|c|c|c|c|}
\hline \multirow[b]{2}{*}{ Genótipo } & \multicolumn{2}{|c|}{------------------ Fase ninfal ------------------- } & \multicolumn{2}{|c|}{---------------------- Fase adulta ---------------' } \\
\hline & Duração (dias) & Mortalidade (\%) & Pré-oviposição (dias) & $\begin{array}{l}\text { Número de } \\
\text { ovos/fêmea }\end{array}$ \\
\hline IAC-BBS 8 & $39,7 \pm 1,02 \mathrm{a}$ & $64 \pm 1,55 \mathrm{a}$ & $9,0 \pm 0,00 \mathrm{a}$ & $21,0 \pm 0,00 \mathrm{a}$ \\
\hline Brachiaria brizantha cv. 'Marandu' & $39,2 \pm 1,06 \mathrm{ab}$ & $66 \pm 1,49 \mathrm{a}$ & $6,0 \pm 0,71 \mathrm{a}$ & $44,5 \pm 14,79 \mathrm{a}$ \\
\hline Cana-de-açúcar cv. 'SP80-1842' & $38,5 \pm 0,43 \mathrm{ab}$ & $22 \pm 0,48 \mathrm{c}$ & $6,0 \pm 0,36 \mathrm{a}$ & $187,0 \pm 29,93 \mathrm{~b}$ \\
\hline BB 39 & $38,2 \pm 1,03 \mathrm{ab}$ & $28 \pm 0,84 \mathrm{bc}$ & $6,7 \pm 0,33$ a & $85,5 \pm 37,07 \mathrm{ab}$ \\
\hline BB 33 & $36,9 \pm 0,13 \mathrm{~b}$ & $26 \pm 0,82 \mathrm{c}$ & $6,1 \pm 0,41 \mathrm{a}$ & $32,9 \pm 9,24 \mathrm{a}$ \\
\hline Setaria anceps cv. 'Kazungula' & $36,8 \pm 0,56 \mathrm{~b}$ & $62 \pm 0,88 \mathrm{a}$ & $6,3 \pm 0,61 \mathrm{a}$ & $36,5 \pm 20,58 \mathrm{a}$ \\
\hline IAC-BBS 5 & $34,5 \pm 0,35 \mathrm{c}$ & $54 \pm 0,48 \mathrm{ab}$ & $6,0 \pm 0,33 \mathrm{a}$ & $21,4 \pm 14,79 \mathrm{a}$ \\
\hline
\end{tabular}

Médias seguidas de mesma letra nas colunas não diferem entre si pelo teste de Tukey $(\mathrm{p}<0,05)$

Ciência Rural, v.44, n.6, jun, 2014. 
significativa $(\mathrm{F}=0,91, \mathrm{p}=0,4967)$ entre as médias dos genótipos (Tabela 1).

A fecundidade foi influenciada pelos diferentes genótipos avaliados, obtendo-se os menores valores (21,0 a 44,5 ovos) em cinco dos sete genótipos testados, os quais diferiram apenas da média registrada em cana-de-açúcar, cv. 'SP80-1842' (187,00 ovos), constatando-se em BB 39 (85,5 ovos) um valor intermediário (Tabela 1). NILAKHE (1987) verificou menor fecundidade para Deois flavopicta (Stål) quando criada em A. gayanus, cv. 'Planaltina', e P. guenoarum; no entanto, para $\boldsymbol{M}$. fimbriolata, não foi observada diferença nesse parâmetro em relação às diversas gramíneas testadas ( $\boldsymbol{P}$. maximum $\mathrm{cv}$. 'Tobiatã', B. decumbens cv. 'Basilisk', B. brizantha cv. 'Marandu', $\boldsymbol{P}$. plicatulum e $\boldsymbol{P}$. guenoarum). SUJII et al. (2001) também registraram menor fecundidade para D. flavopicta quando criada sobre Axonopus marginatus (5,3 ovos), em comparação a $\boldsymbol{B}$. ruziziensis (29,4 ovos), indicando que a fecundidade estaria relacionada com a alimentação dos adultos. Diferenças na fecundidade de $\boldsymbol{M}$. fimbriolata foram relatadas por GARCIA (2006) ao utilizar diversas cultivares de cana-de-açúcar e comprovar a influência da alimentação no número de descendentes, como observado neste trabalho.

Não foi constatado efeito significativo da interação dos fatores genótipo e sexo ( $\mathrm{F}=10,20, \mathrm{p}=0,3453)$ sobre a longevidade de $\boldsymbol{M}$. fimbriolata (Tabela 2). Assim, considerando-se a média entre machos e fêmeas, verificou-se que, independentemente do sexo das cigarrinhas, as longevidades registradas em IAC-BBS 8 (6,6 dias) e IAC-BBS 5 ( 7,4 dias) foram menores $(\mathrm{P}=0,0001)$ que as encontradas em cana-de-açúcar cv. 'SP80-
1842', em que foi registrado o maior valor $(15,2$ dias), e em B. brizantha cv. 'Marandu' (10,2 dias). As longevidades encontradas naqueles genótipos, no entanto, não diferiram das verificadas nos demais genótipos, que propiciaram valores intermediários (7,6 a 9,1 dias). Por outro lado, independentemente do genótipo em que as cigarrinhas foram criadas, as longevidades médias de machos e fêmeas (9,3 e 9,0 dias, respectivamente) não diferiram entre si. Em condições de campo, a redução da longevidade das cigarrinhas é importante, porque isso possivelmente irá diminuir o seu período de oviposição, reduzindo, consequentemente, o crescimento da população do inseto nas próximas gerações. Além disso, a menor longevidade irá diminuir o tempo de permanência dos adultos no campo, reduzindo o grau de injúria causado pelas toxinas injetadas nas plantas durante a alimentação, o que se constitui no principal dano causado por essa praga. NILAKHE (1987) observou longevidade média de 8,2 dias para $\boldsymbol{M}$. fimbriolata em B. brizantha, cv. 'Marandu', apresentando semelhança com os dados obtidos neste trabalho.

Fase embrionária

A viabilidade dos ovos variou de 24,3 a $100 \%$, sendo os menores valores registrados em IACBBS 5 (24,3\%) e em B. brizantha cv. 'Marandu' (47,5\%), diferindo de todos demais genótipos, nos quais os valores variaram entre 78,2 e $100 \%$ (Tabela 3). Não houve diferença na duração da fase embrionária entre os genótipos (Tabela 3), obtendose duração média de 20 dias, com variação de 19,5 a 22,5 dias, ocorrendo a maior frequência de eclosão de ninfas ao $19^{\circ}$ dia.

Tabela 2 - Médias ( \pm erro padrão) de longevidade de adultos de Mahanarva fimbriolata, criada em sete gramíneas $\left(\mathrm{T}=25 \pm 1^{\circ} \mathrm{C}\right.$; $\mathrm{UR}=70 \pm 10 \%$; fotofase $14 \mathrm{~h})$.

\begin{tabular}{|c|c|c|c|}
\hline \multirow[t]{2}{*}{ Genótipo } & \multirow[b]{2}{*}{ Macho } & \multirow[b]{2}{*}{ Fêmea } & \multirow[b]{2}{*}{ Total } \\
\hline & & & \\
\hline IAC-BBS 8 & $6,5 \pm 0,61$ & $6,7 \pm 0,51$ & $6,6 \pm 0,56 \mathrm{a}$ \\
\hline IAC-BBS 5 & $6,7 \pm 0,61$ & $8,1 \pm 0,74$ & $7,4 \pm 0,68 \mathrm{a}$ \\
\hline Setaria anceps cv. 'Kazungula' & $7,2 \pm 0,91$ & $8,0 \pm 0,72$ & $7,6 \pm 0,82 \mathrm{ab}$ \\
\hline BB 33 & $7,9 \pm 0,33$ & $7,5 \pm 0,48$ & $7,7 \pm 0,41 \mathrm{ab}$ \\
\hline BB 39 & $9,3 \pm 1,22$ & $8,9 \pm 1,47$ & $9,1 \pm 1,35 \mathrm{ab}$ \\
\hline Brachiaria brizantha cv. 'Marandu' & $10,9 \pm 0,90$ & $9,6 \pm 1,28$ & $10,2 \pm 1,09 \mathrm{~b}$ \\
\hline Cana-de-açúcar cv. SP80-1842 & $16,3 \pm 1,02$ & $14,1 \pm 1,23$ & $15,2 \pm 1,13 \mathrm{c}$ \\
\hline Média & $9,3 \pm 0,80 \mathrm{~A}$ & $9,0 \pm 0,92 \mathrm{~A}$ & \\
\hline
\end{tabular}

Médias seguidas de mesma letra, minúscula nas colunas e maiúsculas nas linhas, não diferem entre si, pelo teste de Tukey (P<0,05). 
Tabela 3 - Médias ( \pm erro padrão) de viabilidade e duração da fase de ovo de Mahanarva fimbriolata, criada em sete gramíneas $\left(\mathrm{T}=25 \pm 1^{\circ} \mathrm{C} ; \mathrm{UR}=70 \pm 10 \%\right.$; fotofase 14h)

\begin{tabular}{lcl}
\hline & & \\
Genótipo & Viabilidade (\%) & Duração (dias) \\
\hline IAC-BBS 8 & $100,00 \pm 0,00 \mathrm{~d}$ & $19,5 \pm 0,35 \mathrm{a}$ \\
IAC-BBS 5 & $24,3 \pm 10,99 \mathrm{a}$ & $20,0 \pm 0,71 \mathrm{a}$ \\
Setaria anceps cv. & $81,7 \pm 13,80 \mathrm{~cd}$ & $20,0 \pm 0,82 \mathrm{a}$ \\
'Kazungula' & $78,2 \pm 2,31 \mathrm{c}$ & $20,0 \pm 0,31 \mathrm{a}$ \\
BB 33 & $90,2 \pm 3,86 \mathrm{~cd}$ & $22,5 \pm 1,04 \mathrm{a}$ \\
BB 39 & $47,5 \pm 11,35 \mathrm{~b}$ & $22,0 \pm 0,30 \mathrm{a}$ \\
Brachiaria brizantha & & \\
cv. 'Marandu' & & $21,0 \pm 1,15 \mathrm{a}$ \\
Cana-de-açúcar cv. & $95,3 \pm 0,84 \mathrm{~cd}$ & \\
SP80-1842 & & \\
Média & & \\
\hline
\end{tabular}

Médias seguidas de mesma letra, minúscula nas colunas e maiúsculas nas linhas, não diferem entre si, pelo teste de Tukey $(\mathrm{P}<0,05)$.

Discriminação dos genótipos pela análise de agrupamento e tabela de vida de fertilidade

A análise de agrupamento permitiu a formação de três grupos distintos: o primeiro grupo foi formado por IAC-BBS 5 e B. brizantha, cv. 'Marandu' (grupo 1), o segundo por S. anceps, cv. 'Kazungula', IAC-BBS 8 e BB 33 (grupo 2) e o terceiro por BB
39 e cana-de-açúcar (grupo 3) (Figura 1). Entre os parâmetros estudados para $\boldsymbol{M}$. fimbriolata, os que mais influenciaram a formação dos grupos foram provavelmente o número médio de ovos por fêmea e a viabilidade desses ovos, já que, no grupo 3, estão incluídos os genótipos em que ocorreram os maiores valores de fecundidade e de viabilidades de ovos. No grupo 2, estão os genótipos que propiciaram valores intermediários para esses parâmetros e, no grupo 1, aqueles em que esses parâmetros apresentaram os menores valores.

A observação dos dados da tabela de vida de fertilidade permite constatar que não houve diferença da taxa líquida de reprodução (Ro) de M. fimbriolata entre os tratamentos, com exceção daquele observado em cana-de-açúcar, cv. 'SP801842 ', no qual foi registrado o maior valor $(174,06)$ (Tabela 4). Já no que se refere ao intervalo médio entre gerações (T), IAC-BBS 8 foi aquele em que ocorreu o maior valor $(104,00)$, diferindo dos constatados em todos os outros genótipos, os quais não diferiram entre si, variando entre 6,62 e 22,08 (Tabela 4). Os valores da taxa intrínseca de crescimento $\left(\mathrm{r}_{\mathrm{m}}\right)$ foram positivos para todos, indicando que os sete genótipos testados propiciaram o crescimento populacional do inseto. A comparação entre os genótipos, entretanto, permite inferir que, em IAC-BBS 8, ocorreu o menor crescimento populacional $(0,01)$, diferindo de todos os demais, sugerindo que esse material apresenta

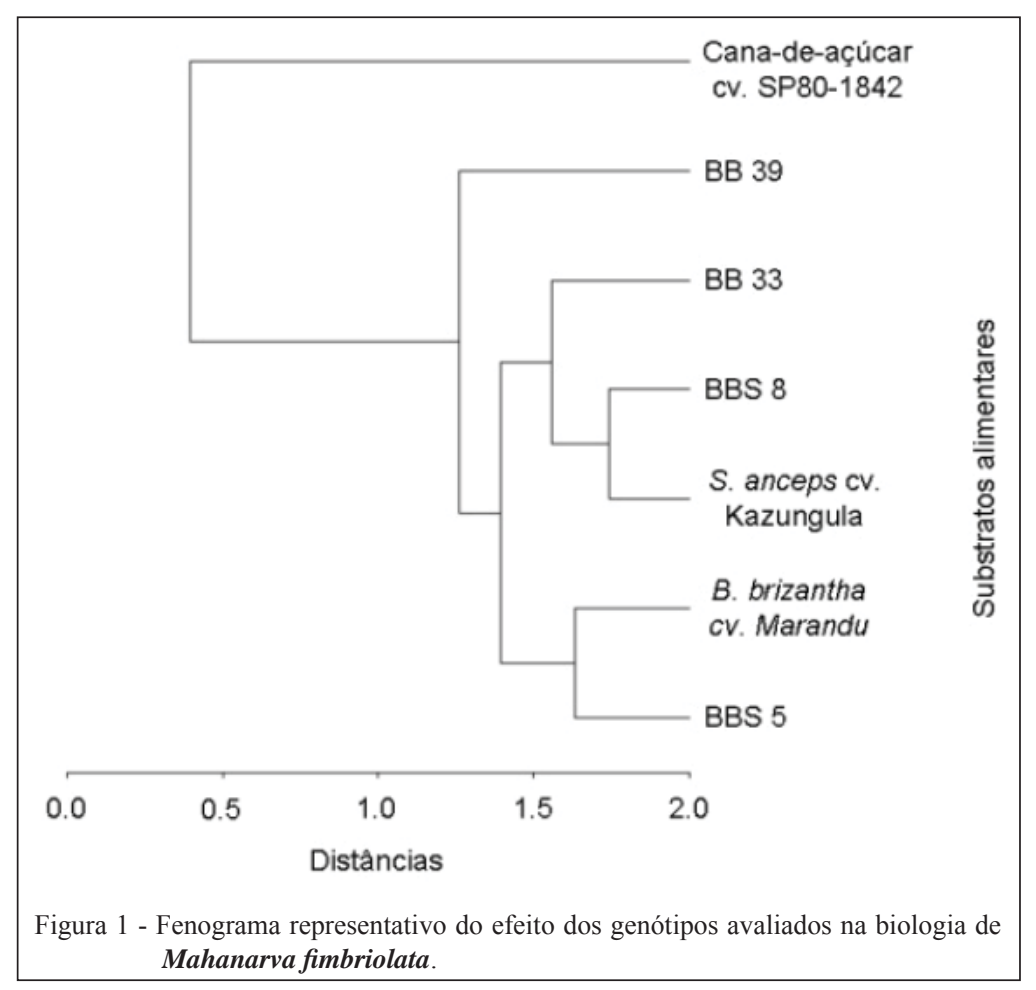

Ciência Rural, v.44, n.6, jun, 2014. 
Tabela 4 - Estimativas dos parâmetros associados às tabelas de vida de fertilidade \pm erro padrão (E.P.) de Mahanarva fimbriolata e valores $\mathrm{p}$ correspondentes ao teste $\mathrm{t}$, para comparação entre pares de tratamentos, utilizando variâncias estimadas pelo método Jackknife.

\begin{tabular}{|c|c|c|}
\hline Parâmetro & Genótipo & Média \pm E.P. \\
\hline \multirow{7}{*}{ Ro } & IAC-BBS 8 & $1,37 \pm 1,37 \mathrm{a}$ \\
\hline & IAC-BBS 5 & $3,91 \pm 2,19 \mathrm{a}$ \\
\hline & $\begin{array}{l}\text { Brachiaria brizantha, } \\
\text { cv. 'Marandu' }\end{array}$ & $9,58 \pm 4,42 \mathrm{a}$ \\
\hline & $\begin{array}{l}\text { Setaria anceps, cv. } \\
\text { 'Kazungula' }\end{array}$ & $14,67 \pm 9,05 \mathrm{a}$ \\
\hline & BB 33 & $15,32 \pm 5,21 \mathrm{a}$ \\
\hline & BB 39 & $20,24 \pm 10,64 \mathrm{a}$ \\
\hline & $\begin{array}{l}\text { Cana-de-açúcar, cv. } \\
\text { 'SP80-1842' }\end{array}$ & $174,06 \pm 27,75 b$ \\
\hline \multirow{7}{*}{$\mathrm{T}$} & IAC-BBS 8 & $104,00 \pm 0,00 \mathrm{a}$ \\
\hline & IAC-BBS 5 & $22,08 \pm 23,51 \mathrm{~b}$ \\
\hline & $\begin{array}{l}\text { B. brizantha, cv. } \\
\text { 'Marandu' }\end{array}$ & $14,46 \pm 3,68 b$ \\
\hline & $\begin{array}{l}\text { S. anceps, cv. } \\
\text { 'Kazungula' }\end{array}$ & $11,38 \pm 5,72 \mathrm{~b}$ \\
\hline & BB 33 & $11,19 \pm 1,62 \mathrm{~b}$ \\
\hline & BB 39 & $11,06 \pm 2,49 \mathrm{~b}$ \\
\hline & $\begin{array}{l}\text { Cana-de-açúcar, cv. } \\
\text { 'SP80-1842' }\end{array}$ & $6,62 \pm 0,20 \mathrm{~b}$ \\
\hline \multirow{7}{*}{$\mathrm{Rm}$} & IAC-BBS 8 & $0,01 \pm 0,00 \mathrm{a}$ \\
\hline & IAC-BBS 5 & $0,03 \pm 0,02 \mathrm{~b}$ \\
\hline & $\begin{array}{l}\text { B. brizantha, cv. } \\
\text { 'Marandu' }\end{array}$ & $0,05 \pm 0,01 \mathrm{~b}$ \\
\hline & BB 33 & $0,06 \pm 0,01 \mathrm{~b}$ \\
\hline & BB 39 & $0,06 \pm 0,01 \mathrm{~b}$ \\
\hline & $\begin{array}{l}\text { S. anceps, cv. } \\
\text { 'Kazungula' }\end{array}$ & $0,06 \pm 0,02 \mathrm{bc}$ \\
\hline & $\begin{array}{l}\text { Cana-de-açúcar, cv. } \\
\text { 'SP80-1842' }\end{array}$ & $0,10 \pm 0,00 \mathrm{c}$ \\
\hline \multirow{7}{*}{$\Lambda$} & IAC-BBS 8 & $1,01 \pm 0,00 \mathrm{a}$ \\
\hline & IAC-BBS 5 & $1,03 \pm 0,02 \mathrm{~b}$ \\
\hline & $\begin{array}{l}\text { B. brizantha, cv. } \\
\text { 'Marandu' }\end{array}$ & $1,05 \pm 0,01 \mathrm{~b}$ \\
\hline & BB 33 & $1,06 \pm 0,01 \mathrm{~b}$ \\
\hline & BB 39 & $1,07 \pm 0,01 \mathrm{~b}$ \\
\hline & $\begin{array}{l}\text { S. anceps, cv. } \\
\text { 'Kazungula' }\end{array}$ & $1,07 \pm 0,02 \mathrm{bc}$ \\
\hline & $\begin{array}{l}\text { Cana-de-açúcar, cv. } \\
\text { 'SP80-1842' }\end{array}$ & $1,11 \pm 0,00 \mathrm{c}$ \\
\hline
\end{tabular}

Médias seguidas da mesma letra na coluna, para cada parâmetro, não diferem entre si pelo teste " $\mathrm{t}$ " $(\mathrm{P} \leq 0,05)$.

resistência ao inseto. A cana-de-açúcar proporcionou o maior crescimento $(0,10)$, não diferindo apenas de S. anceps cv. 'Kazungula' $(0,06)$ (Tabela 4).
A razão finita de aumento $(\lambda)$, responsável pela indicação do número de fêmeas que são adicionadas à população por cada fêmea, apresentou diferenças significativas entre os genótipos. Para IAC-BBS 8, foi registrado o menor valor $(1,01)$, que diferiu de todos os demais. Os parâmetros avaliados através da tabela de vida de fertilidade demonstraram diferenças no comportamento da praga quando criada sobre os diferentes genótipos. O principal parâmetro para avaliar a qualidade de um substrato alimentar para o desenvolvimento do inseto é a taxa líquida de reprodução; neste trabalho, registrou-se grande diferença entre a testemunha (cana-de-açúcar) e os demais genótipos testados, que se mostraram menos favoráveis ao desenvolvimento de $\boldsymbol{M}$. fimbriolata.

\section{CONCLUSÃO}

As gramíneas IAC-BBS 8 e $\boldsymbol{B}$. brizantha, cv. 'Marandu', apresentam os maiores graus de resistência a $\boldsymbol{M}$. fimbriolata, do tipo antibiose e/ou não preferência para alimentação. As gramíneas BB 33 e BB 39 apresentam suscetibilidade a essa cigarrinha.

\section{REFERÊNCIAS}

AUAD, A.M. et al. Seleção de genótipos de capim-elefante quanto à resistência à cigarrinha-das-pastagens. Pesquisa Agropecuária Brasileira, Brasília, v.42, n.8, p.1077-1081, 2007. Disponível em: $<$ http://www.scielo.br/pdf/pab/v42n8/a03v42n8.pdf $>$ Acesso em: 15 out 2012. doi: 10.1590/S0100-204X2007000800003

BYERS, R.A.; WELLS, H.D. Phytotoxemia of Coastal bermudagrass caused by the two-lined spittlebug Prosapia bicincta (Homoptera: Cercopidae). Annals of the Entomological Society of America, Columbus, v.59, n.6, p.1067-1071, 1966.

FERRUFINO, A.; LAPOINTE, S.L. Host plant resistance em Brachiaria grasses to the spittlebug Zulia colombiana. Entomologia Experimentalis et Applicata, Amsterdam, v.51, p.155-162, 1989. Disponível em: <http://onlinelibrary.wiley.com/ doi/10.1111/j.1570-7458.1989.tb01227.x/abstract> Acesso em: 14 abr 2012. doi: 10.1111/j.1570-7458.1989.tb01227.x.

GARCIA, J.F. Bioecologia e manejo da cigarrinha-das-raízes, Mahanarva fimbriolata (Stål, 1854) (Hemiptera: Cercopidae) em cana-de-açúcar. 2006. 99f. (Doutorado em Entomologia) Escola Superior de Agricultura "Luiz de Queiroz", Universidade de São Paulo, Piracicaba, SP.

GARCIA, J.F. et al. Laboratory rearing technique of Mahanarva fimbriolata (Stål) (Hemiptera: Cercopidae). Scientia Agricola, v.64, n.1, p.73-76, 2007. Disponível em: <http://www.scielo. br/scielo.php?script $=$ sci_arttext\&pid $=\mathrm{S} 010390162007000100$ 011\&lng=en\&nrm=iso>. doi: http://dx.doi.org/10.1590/S010390162007000100011 . 
LAPOINTE, S.L. et al. A methodology for evaluation of host plant resistance in Brachiaria to spittlebug species (Homoptera: Cercopidae). In: INTERNATIONAL GRASSLAND CONGRESS, 16., 1989, Nice France. Proceedings... Nice, France: INRA, 1989. p.731-732.

LAPOINTE, S.L. et al. Antibiosis to spittlebugs (Homoptera: Cercopidae) in accessions of Brachiaria spp. Journal of Economic Entomology, v.85, n.4, p.1485-1490, 1992.

MAIA, H.N.M. et al. Statistical inference on associated fertility life table parameters using jackknife technique: computational aspects. Journal of Economic Entomology, v.93, p.511-518, 2000 Disponível em: <http://www.bioone.org/doi/abs/10.1603/00220493-93.2.511? $>$ journal Code=ecen. doi: http://dx.doi. org/10.1603/0022-0493-93.2.511

MEYER, J.S. et al. Estimating uncertainly in population growth rates: jackknife vs. bootstrap techniques. Ecology, v.67, p.11561166, 1986. Disponível em: <http://www.jstor.org/stable/ 1938671>. Acesso em: 17 ago 2012.

NILAKHE, S.S. Evaluation of grasses for resistance to spittlebugs. Pesquisa Agropecuária Brasileira, v.22, n.8, p.767-783, 1987.
SILVEIRA NETO, S. et al. Manual de ecologia dos insetos. São Paulo: Ceres, 1976. 419p.

SUJII, E.R. et al. Effect of host plant on the fecundity of spittlebug Deois flavopicta Stal (Homoptera: Cercopidae): implications on population dynamics. Neotropical Entomology, v.30, n.4, p.547552, 2001. Disponível em: <http://www.scielo.br/scielo.php? script=sci_arttext\&pid=S1519-566X2001000400006\&lng=en\&nr $\mathrm{m}=\mathrm{iso}>$. Acesso em: 23 Jan 201. doi: http://dx.doi.org/10.1590/ S1519-566X2001000400006.

VALÉRIO, J.R. Caracterização de dano causado pelo adulto da cigarrinha-das-pastagens Zulia entreriana (Berg, 1879) em Brachiaria decumbens Stapf, cv. 'Basilisk'. 1985. 152f. Tese (Doutorado em Entomologia) - ESALQ, Piracicaba, SP.

VALÉRIO, J.R. Considerações sobre a morte de pastagens de Brachiaria brizantha cv. 'Marandu' em alguns Estados do Centro e Norte do país - Enfoque entomológico. Campo Grande: Embrapa Gado de Corte, 2006. Comunicado técnico 98. p1-8. Disponível em: $<$ http://www.infoteca.cnptia.embrapa.br/bitstream/ doc/326986/1/COT98.pdf>. Acesso 06 nov 2012.

WILKINSON, L. Systat for windows. Evanston: Systat, 1992. 547p. 